\title{
Can Self-Doubt Be Beneficial to Performance? Exploring the Concept of Preparatory Efficacy
}

\author{
Deborah L. Feltz* and Jared M. Wood \\ Department of Kinesiology, Michigan State University, East Lansing, MI 48824, USA
}

\begin{abstract}
Sport competition can be divided into a preparatory (practice) and performance (competition) process. Selfefficacy beliefs taken just prior (i.e., within $24 \mathrm{hr}$ or after a final practice session) to the actual competition are referred to as performance efficacy beliefs; whereas, efficacy beliefs measured during the preparation or practice stage are referred to as preparatory efficacy beliefs. Consistent with the vast majority of efficacy research, Bandura [1] suggests that high performance efficacy perceptions are best for performance. In contrast, he suggests that preparatory efficacy perceptions should optimally reflect some sense of self-doubt because they serve as an impetus that motivates increased preparatory effort. Ultimately, greater preparatory effort should lead to stronger competitive performance. Despite Bandura's observations of this process at work, no empirical studies have examined preparatory efficacy perceptions, preparatory effort, performance efficacy, and performance across a single preparation-competition process. This article examines the concept of preparatory efficacy, existing experiential and empirical support for the concept, and finally, suggestions, applications, and implications for future research.
\end{abstract}

\section{CAN SELF-DOUBT BE BENEFICIAL TO PER- FORMANCE? EXPLORING THE CONCEPT OF PREPARATORY EFFICACY}

In self-efficacy theory applied to sports, Bandura [1] distinguishes between the concepts of preparatory efficacy and performance efficacy. Although both are measures of one's self-efficacy regarding an upcoming athletic performance, as Feltz, Short, and Sullivan [2] note, the concepts differ in timing of measurement. Preparatory efficacy is measured during the preparation (i.e. practice) phase of competition [1]; whereas, performance efficacy is measured immediately prior to, or as close as possible to, the start of the actual competitive performance (see Fig. 1). Thus far, most research has used performance efficacy as the selfefficacy measure or has failed to differentiate between the two concepts. As Bandura argues, this may be an important oversight, because the timing of the efficacy measurement may have important implications for proximal behavior. The remainder of this article addresses a brief overview of what is known about performance efficacy, the differences between performance efficacy and preparatory efficacy, existing evidence of preparatory efficacy, and considerations for research on the concept.

\section{OVERVIEW AND CURRENT CONSENSUS ON SELF- EFFICACY AND SPORT PERFORMANCE}

This overview of performance efficacy is not intended to be comprehensive. Rather, this overview focuses on what is known about performance efficacy in order to highlight the proposed differences, between performance efficacy and preparatory efficacy. For a comprehensive review of self-efficacy research in sport, see Feltz et al. [2].

\footnotetext{
*Address correspondence to this author at the Department of Kinesiology, Michigan State University, East Lansing, MI 48824, USA; Tel: (517) 3554732; Fax: (517) 353-2944; E-mail: dfeltz@msu.edu
}

An efficacy judgment is one's perception of what one can do under specified conditions. Efficacy beliefs have been studied at the individual level and group level (termed collective efficacy) [1]. In sports, performance efficacy is the judgment of an athlete's capability, or his or her team's capability, in performing at a certain level or successfully executing certain sport-related attainments under given conditions. Performance efficacy has been demonstrated to have a moderate positive, linear relationship with performance, particularly when the efficacy measurement and performance are concordant [3]. Given the numerous possible factors that influence performance, the magnitude of this relationship has been deemed ample and meaningful by researchers in the field [2].

Efficacy beliefs are changeable and are influenced by various sources of information. Sources of self-efficacy include past performances, vicarious learning experiences (i.e. modeling), verbal persuasion, and physical and affective states [1]. Feltz et al. [2] noted that two additional categories of efficacy sources have been included in the research: emotional states and imaginal experiences.

The efficacy-performance relationship over time has been shown to be temporally recursive [1]. Within sport tasks, studies have demonstrated that efficacy has a causal effect on performance in laboratory studies, in which efficacy beliefs are manipulated by nonperformance means, such as through modeling, imagery, and bogus norms [e.g., $4,5,6]$. Others have shown that performance has a causal effect on efficacy [7]. Additionally, Feltz, Chow, and Hepler [8] showed a reciprocal relationship between self-efficacy and performance across four trials of diving performance when controlling for past performance and past efficacy beliefs. Myers, Payment, and Feltz [9] have further demonstrated, using a within team analysis, that, across a competitive season, collective efficacy has an impact on performance, and performance has a subsequent influence on collec- 


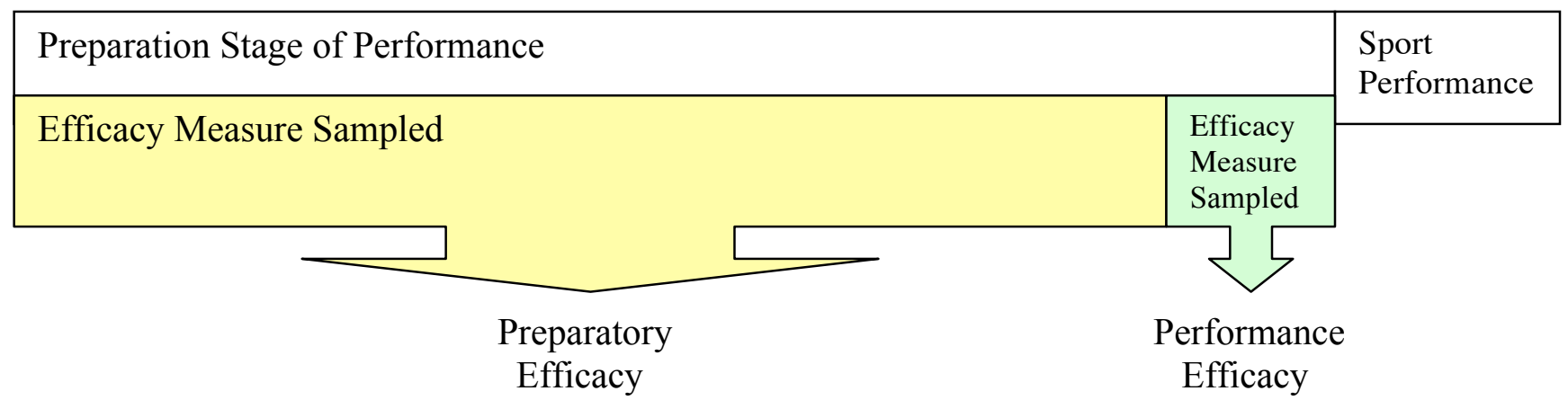

Fig. (1). Stages of sport performance and corresponding efficacy measures sampled during each stage.

tive efficacy. Thus, the temporally recursive nature of the efficacy-performance relationship has been demonstrated in both laboratory and field settings, using both between subjects and within subjects designs.

Efficacy influences performance through its effect on choice, effort, and persistence. Efficacious individuals choose to engage in harder tasks, expend more effort in their chosen activities, and persist longer when faced with difficulty. The Feltz et al. [2] review of the self-efficacy research in sport further supports the idea that self-efficacy tends to be beneficial for goal setting, self-regulation, attributions for performance, decision making, optimistic and pessimistic thinking, anxiety regulation, and fear and worry reduction.

\section{PREPARATORY EFFICACY AS A CONSTRUCT}

Bandura [1] makes this distinction because self-efficacy beliefs at various stages of a preparation-competition cycle are likely to have implications for proximal behaviors. Given the mutual influences of efficacy beliefs on performance and performance on efficacy beliefs, the faster, higher, stronger mentality of competitive sports has tended to view high efficacy as the optimum state for performance (see Fig. 2). Accordingly, Bandura has argued that performance efficacy should be as high as possible, given, of course, that one has the relevant skills to perform. As he describes it, gametime performance of learned skills is difficult when one is wrestling with self-doubt (i.e. when performance efficacy is low); therefore, performance is best executed when an athlete is in an efficacious frame of mind. In contrast, however, he suggests that preparatory efficacy should be relatively lower than one's performance efficacy so that it serves as an impetus to summon greater effort during practice for competition (see Fig. 3). Preparatory effort seems unnecessary if athletes are complacently selfconfident about their ability to perform well in an upcoming contest (i.e. when preparatory efficacy is high). Thus, Bandura reasons that some sense of self-doubt is desirable in preparatory efficacy levels because it motivates one to expend greater preparatory effort and focus, which will ultimately result in greater skill development and execution. As he states, "In short, self-doubt creates the impetus for acquiring knowledge and skills, but it hinders proficient use of developed skills." (p.76). The basis of Bandura's theory of preparatory efficacy is that high efficacy hinders learning of skills (i.e., practice and perfection of skills in sports), but is beneficial to application of skills (i.e., performance in competition). Thus, Feltz et al. [2] suggest that Bandura's hypothesis implies a curvilinear, or inverted-U relationship in the preparatory phase between self-efficacy beliefs and effort and persistence as the dependent variable.

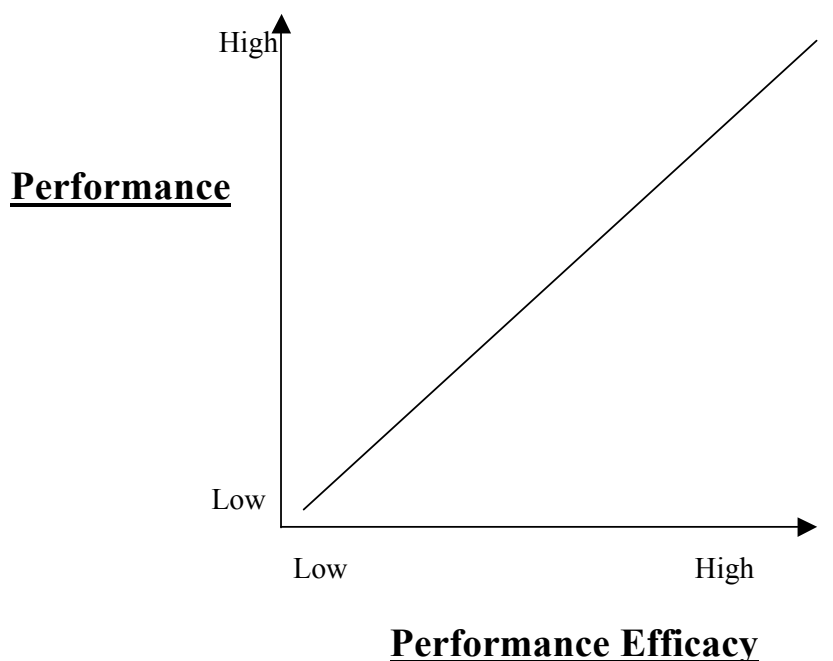

Fig. (2). The relationship between performance efficacy and performance level.

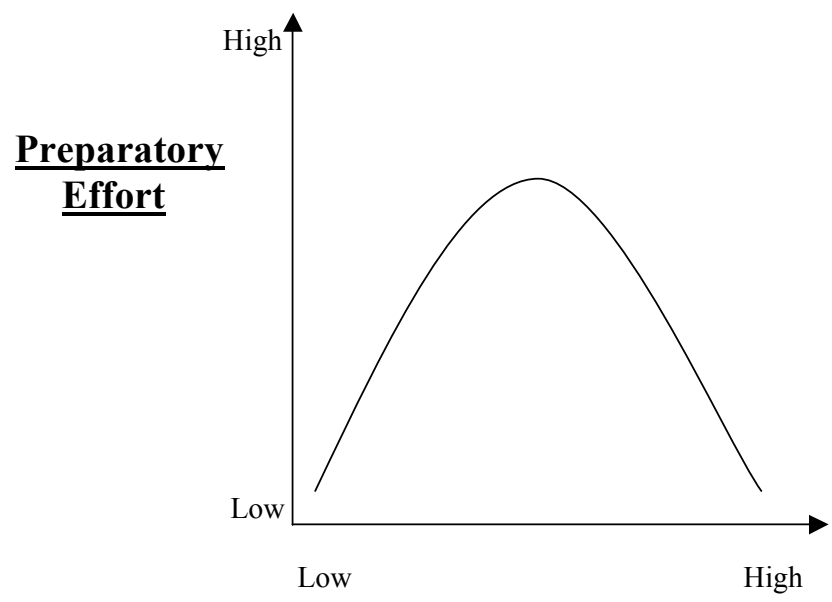

Preparatory Efficacy

Fig. (3). The proposed relationship between preparatory efficacy and preparatory effort. 
To be sure, Bandura notes that doubt only enhances effort when a threshold of efficacy already exists. Without this minimal level of efficacy, athletes may simply succumb to abject doubt and slacken effort in order to protect selfesteem through defensive pessimism.

Bandura [1] is careful to maintain that coaches who attenuate preparatory efficacy levels should be heedful of restoring or increasing efficacy levels before a competition (see Fig. 4). Although he does not mention a specific pathway for increasing efficacy beliefs from the preparatory stage, the authors of this article contend that when greater preparatory effort is expended the availability of efficacy enhancing information (i.e. favorable mastery experiences, vicarious experiences, verbal persuasion, and physiological and affective states) should increase, and through the interplay of coach and athlete, a strong sense of efficacy can be restored before the competitive performance.

Despite the encouraging idea that a sense of self-doubt, normally considered a detriment to athletic performance, could be beneficial under certain circumstances, no adequate empirical studies have supported this concept. The following review of empirical studies and experiential observations is offered to help illustrate how the concept of preparatory efficacy may work in a sport setting. The review of evidence is not offered in an attempt to validate a concept that clearly needs further study. Rather, we offer it to argue that sport psychologists need a more detailed picture of the efficacyperformance relationship across a preparation-competition process.

\section{ANECDOTAL EVIDENCE OF PREPARATORY EFFICACY}

In turning to experiential support for preparatory efficacy, it is important to note that we do not consider the following examples to be the only experiential support we have encountered for preparatory efficacy as Bandura has described it; however, we consider these few examples to be important accounts of sport phenomena that are not parsimoniously explained by our current understanding of self-efficacy. With that, we turn to Bandura's [1] observations that coaches frequently apply the preparatory efficacy concept to their craft. He has stated that coaches often exaggerate the strengths of opponents while emphasizing their own athletes' weaknesses in order to reduce preparatory efficacy levels. Presumably, this helps motivate them to give a greater preparatory effort than they would have given under high efficacy conditions. Before the beginning of the performance phase, though, coaches take care to build up player efficacy levels via positive feedback and verbal persuasive techniques in order to send their athletes into a contest with a restored or increased sense of efficacy.

Anecdotally, Seligman [10] wrote about an informal quasi-experiment in which coaches reported worse than expected baseline event times to a group of elite swimmers who had known scores on a test of optimism-pessimism. Although no efficacy perceptions were measured, the purpose of their false reporting was to create a sense of doubt in the swimmers' efficacy beliefs regarding their event time. Following the manipulation of the reported swim times, the sense of initial doubt that the swimmers experienced was transformed by the optimists into faster times in the next practice trial. The pessimists did not experience a similar improvement in performance. Their selfdoubt was defeating. Seligman used the optimism-pessimism continuum to explain his findings, but an explanation that takes into consideration Bandura's concept of preparatory efficacy, in which efficacy reducing information is translated into a better effort in the next practice trial, is easily transposed onto Seligman's explanation. Certainly, this example

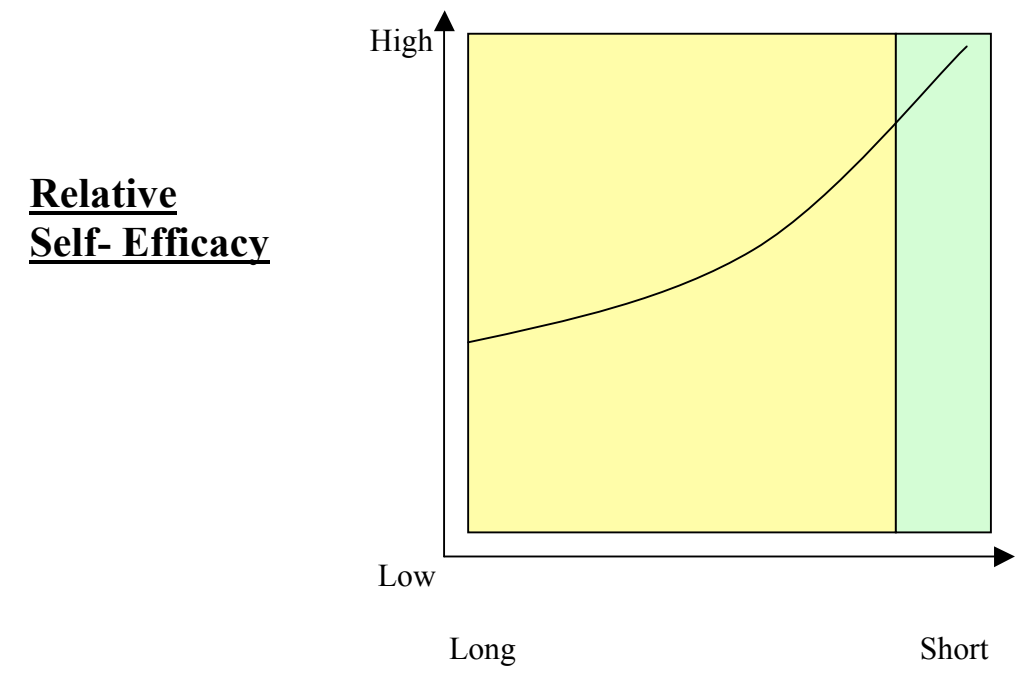

Type of Self-Efficacy Sampled:

$\underline{\text { Relative Time to Performance }}$

Preparatory Efficacy: $\square$
Performance Efficacy: $\square$

Fig. (4). The proposed optimal curve for relative self-efficacy levels in comparison to relative time to performance. 
fits with Bandura's observations and predictions of the active attempts of coaches to regulate their athletes' efficacy beliefs in order to influence effort in proximal behavior. Further, even though the swimmers were timed in their events, the setting was obviously a practice situation. Thus, this example took place entirely during the preparation of phase of performance. It is an example of athletes receiving efficacy-reducing information followed by a subsequent increase in their effort toward the task at hand.

Further experiential support for preparatory efficacy exists in the similar concept of overconfidence. Overconfidence is a common term used to explain the reasons behind a favored team's lackluster performance against an underdog opponent. Conventional wisdom implies that the favored team's overconfidence somehow reduces their effort in preparation and/or performance. Weinberg and Gould [11] depict the relationship between overconfidence and performance in competition in an inverted- $U$ shape; however, they use a skewed $U$ to suggest that optimal confidence is within the high confidence range but slightly lower than the highest levels of confidence at which overconfidence can occur. As an explanation for how overconfidence may influence performance in competition, Weinberg and Gould suggest that overconfidence may harm competitive performance when athletes slacken their preparatory efforts in practice. Despite their assertions about overconfidence, Weinberg and Gould did not cite any studies that support the inverted $U$ relationship between confidence (i.e., selfefficacy) and competitive performance nor preparatory practice. Similar to Bandura's observation of coaches' social regulation of preparatory efficacy levels, Weinberg and Gould based their descriptions of overconfidence on years of observation and experience.

Thus, across three expert sources, we have a confluence of opinion suggesting that complacency may occur as the result of overconfidence exhibited by athletes working less hard and giving less effort toward preparation. Essentially, the noted observations of Weinberg and Gould [11] give credence to Bandura's preparatory efficacy concept on three points. First, high confidence (or self-efficacy in Bandura's case) is important for high level performance. Second, at some point between practice trials and competitions, high levels of confidence may, at times, be detrimental to competitive performance. Third, when confidence is detrimental to competitive performance, the harmful effects seem to be related to poor effort, choices, or persistence during the preparation/practice stage of performance.

\section{SELF-EFFICACY STUDIES AND SELF-DOUBT}

Several studies outside the realm of sports have found that a sense of self-doubt, represented by a comparatively lower or moderate sense of self-efficacy, is beneficial to preparatory effort, performance, or both. Salomon [12] found that students invested more cognitive effort and learned more from a task that they considered difficult in comparison to one they considered easy. He concluded that some sense of self-doubt, which resulted in relatively lower efficacy levels, served as information that a greater degree of effort was needed to master the task. Bandura and Jourden [13] observed that a high efficacy group of participants set less lofty goals than a lower efficacy comparison group of participants who struggled to achieve mastery, and over time, the condition of high efficacy appeared detrimental to later performance of a complex decision- making task in a simulated manufacturing business. Along similar lines, Stone [14] found that participants who were induced to have high efficacy on a complex cognitive task did not increase effort, attention, or performance relative to those who were induced to have mildly negative and strongly negative efficacy expectations. Furthermore, participants who were induced to have mildly negative efficacy expectations showed increases in effort, attention, and, subsequently, performance relative to the strongly negative group.

In contrast to the previously mentioned studies of learning and decision making tasks, Eyal, Bar-Eli, Tenebaum, and Pie [15] studied efficacy levels in regard to a motor learning task. Their experiment found that participants performed better on various motor tasks when manipulated self-efficacy was at a moderate level in comparison to either a high or low level. Eyal et al. were not studying preparatory efficacy levels, however, and did not take adequate measures to support the concept. Still, the task was novel and required learning and effort to perform well. The importance of their study lies in the highest performance being achieved by the moderate efficacy group, which was contrary to the hypothesized results that were based on theory and previous research.

In sum, these studies give credence to the idea that a sense of self-doubt may be beneficial to effort in practice and ultimately in performance; however, none of these studies distinguishes a clear separation between preparatory and performance efficacy. Taken together, they are not adequate for supporting preparatory efficacy as a concept in any setting let alone in competitive athletics. Clearly, exploratory and confirmatory research is needed in this area.

\section{RESEARCH CONSIDERATIONS}

Despite some interesting experiential observation and several learning task studies that support preparatory efficacy, a lack of empirical evidence exists to support its validity in sport competition. This is especially troublesome because Bandura's [1] discussion of preparatory efficacy largely revolves around sport performance and coaches' social regulation of player efficacy levels. For sport psychologists to support the validity and utility of preparatory efficacy as a concept, empirical evidence to support three processes must be found. First, if preparatory efficacy is a valid and useful concept, empirical evidence should support the notion that relatively lower preparatory efficacy levels result in increased preparatory effort. Second, relatively lower preparatory efficacy levels should ultimately result in relatively higher competitive performance because of greater preparatory effort. Third, relatively lower preparatory efficacy levels should show increases across time and result in relatively higher performance efficacy as one gets closer to competition. Laboratory studies designed to answer these questions would give the most reliable confirmation of this concept. At this point, we are unaware of any studies that adequately address these issues in any setting, let alone a sport setting.

When conducting preparatory efficacy research, measures of effort and performance are important considerations. It also is important to have concordance between measures 
of efficacy and performance [3]. However, with preparatory efficacy, the efficacy measure is focused on performing the competitive task while the dependent measure is focused on effort. Thus, there is not concordance in the same way as there should be in measuring performance efficacy. Rather than having concordance between efficacy and performance, there must be a mechanically plausible connection between the efficacy measurement and the effort measurement. The effort measurement must be based on a task that has a measureable effort component, and the effort component must be plausibly connected to performance. For example, in the Feltz dive studies [16, 17], if practice dives were allowed, the number of practice dives taken should correlate with performance: The more practice dives taken, the better the performance. If a clear separation of preparation and performance dives was established, the number of practice dives taken of one's own volition could serve as a preparatory effort measure. Likewise, finding the correct pace to a golf putt (i.e. putting to a target rather than a hole) is a task that should benefit from multiple trials. Of course, the correct pace could be found in one putt, but to become consistent, in general, a competitive golfer would benefit from a larger number of practice putts that would give him or her feedback on how much force to apply to have the ball roll the correct distance. Therefore, the number of practice trials taken to establish the correct pace of a putt is an effort measure that is plausibly connected to eventual putting performance. As an example of effort that is not plausibly connected to performance, time on task is a measure of effort that is ambiguously related to performance. Unless it can be shown that the time spent on task is quality time necessary for strong performance, time on task should not be used as an effort measure. The same would be true for any situation in which overtraining was possible. Therefore, many endurance or strength tasks, which lend themselves easily to effort measurements, would not be suitable for preparatory efficacy research because athletes might hold back effort in fear of overtraining effects that could harm their performance.

In conjunction with laboratory research, researchers need evidence supporting the function of preparatory efficacy in applied sport settings. Interviews and observations of coaching behavior could be used to support Bandura's observations of the social management of efficacy levels. Further, interviews and observations of athlete behavior would shed light on how players react to coaches' behaviors. These types of field studies could supplement information gathered in laboratory settings. The confluence or disparity of empirical observations could be used to formulate intervention recommendations, which could lead to studies of intervention effectiveness, an objective of sport psychology that is too often overlooked.

Regardless of the method of inquiry, many meaningful questions about preparatory efficacy deserve answers. For example, do field observations reveal that coaches use techniques for lowering preparatory efficacy levels of their players and if so, do the techniques improve preparatory effort? Is the concept of preparatory efficacy most useful at certain levels of competition (i.e., beginning, intermediate, elite)? Is a certain minimum level or strength of self-efficacy necessary before self-doubt becomes motivating instead of defeating? Why does self-doubt mobilize effort toward preparation? How can a manipulation of self-doubt safely be individualized toward certain athletes? Are certain personality traits or states more inclined to benefit from self-doubt during preparation? What types of interventions are best for safely manipulating efficacy levels to the proper levels at the proper stages of competition? Do the unique skill characteristics of the sport, such as the use of gross vs. fine motor skills or broad-narrow vs. internal-external focus or individual vs. group task, make a difference in whether self-doubt is beneficial to preparatory effort and ultimate performance?

Of course, the same important measurement and design considerations that apply to laboratory research apply to fieldwork. Additionally, work with athletes in their competitive environment involves important considerations that may not be present in the laboratory. When conducting research with actual teams or individual athletes, it is of utmost importance to design studies that do not interfere with the normal preparation and competition processes (e.g. the experimental manipulation of efficacy levels is not ethically responsible behavior for research with athletes in the field). This is a formidable obstacle when the additional obligation of obtaining reliable, valid, and timely measurement is considered.

\section{CONCLUSION}

Research on the preparation-competition cycle can only enhance our understanding of sport as a process. By studying this process, we will gain a more meaningful understanding of athletes' efficacy perceptions and other thoughts, affective states, and behaviors, across the preparation process. Given that in all sports the preparation process is a much greater portion of time than the actual competitive performance, this is an important step in sport psychology research. In learning more about changing efficacy levels across the preparationperformance process, sport psychology professionals are likely to gain a powerful tool for designing new efficacy modulating interventions within the known sources of efficacy (i.e. enactive mastery experiences, vicarious learning experiences, verbal persuasion, and physical states). Whereas in the past, sport psychologists have had very good reasons for learning how to apply efficacy enhancing interventions, research on preparatory efficacy is likely to give us more confidence, regarding how and when to apply efficacy enhancing interventions in relation to efficacy attenuating interventions. As an additional benefit of almost any line of inquiry, initial findings may lead to more questions than answers. Related avenues of study in areas such as affective states, personality traits, attitudes, persuasion, learning, and physiology may be found following a more detailed picture of efficacy levels across a process of preparation and performance cycles.

Perhaps most importantly, by gaining a greater understanding of efficacy across the preparation-competition process, we are likely to learn more about optimal practices for preparing athletes for competition. Between the start of preparation and the end of competition, a rich tapestry of interwoven factors influence performance, not the least of which is the dimension of self-efficacy. Although we know that perceptions of one's capabilities are important, we know relatively little about how one manages the battle between 
self-doubt and self-efficaciousness, and we certainly do not know how differences in self-efficacy perceptions influence behavior at different points in the preparation-competition process. Ultimately, we may learn not to fear self-doubt but to recognize it as a normally occurring opportunity for growth and improved performance.

\section{ACKNOWLEDGEMENTS}

The authors wish to thank Graig Chow and Theresa Hepler for their comments on an earlier draft of this manuscript.

\section{REFERENCES}

[1] Bandura A. Self-efficacy. The Exercise of Control. New York, NY: W.H. Freeman and Company 1997.

[2] Feltz DL, Short SE, Sullivan PJ. Self-efficacy in Sport. Champaign, IL: Human Kinetics 2008.

[3] Moritz SE, Feltz DL, Fahrbach KR, Mack DE. The relation of self-efficacy measures to sport performance: a meta-analytic review. Res Q Exercise Sport 2000; 71: 280-94.

[4] Feltz DL, Reissinger CA. Effects on in vivo emotive imagery and performance feedback on self-efficacy and muscular endurance. $\mathbf{J}$ Sport Exerc Psychol 1990; 12; 132-43.

[5] Weinberg RS, Gould D, Jackson A. Expectations and performance: an empirical test of Bandura's self-efficacy theory. J Sport Psychol $1979 ; 1: 320-31$.

[6] Weinberg RS, Gould D, Yukelson D, Jackson A. The effects of pre-existing and manipulated self-efficacy on a competitive muscular endurance task. J Sport Psychol 1981; 3: 345-54.
[7] Escarti A, Guzman JF. Effects of feedback on self-efficacy, performance, and choice on an athletic task. J Appl Sport Psychol 1999; 1: 83-96.

[8] Feltz DL, Chow GM, Hepler TJ. Path analysis of self-efficacy and diving performance revisited. J Sport Exerc Psychol 2008; 30: 401-11.

[9] Myers ND, Payment CA, Feltz DL. Reciprocal relationships between collective efficacy and team performance in women's ice hockey. Group Dyn-Theor Res 2004; 8: 182-95.

[10] Seligman M P. Learned Optimism: How to Change your Mind and your Life. New York, NY: Pocket Books 1998.

[11] Weinberg RS, Gould D. Foundations of Sport and Exercise Psychology, 4th ed. Champaign, IL: Human Kinetics 2007.

[12] Salomon G. Television is easy and print is tough: the differential investment of mental effort in learning as a function of perceptions and attributions. J Educ Psychol 1984; 76: 647-58.

[13] Bandura A., Jourdan, FJ. 1991. Self-regulatory mechanisms governing the impact of social comparison on complex decision making. J Pers Soc Psychol 1991; 60: 941-51.

[14] Stone D. Overconfidence in initial self-efficacy judgments: Effects on decision processes and performance. Organ Behav Hum Decis Process 1994; 59: 452-74.

[15] Eyal N, Bar-Eli M, Tenenbaum G, Pie JS. Manipulated outcome expectations and competitive performance in motor tasks with gradually increasing difficulty. Sport Psychol 1995; 9: 188-200.

[16] Feltz DL. A path analysis of the causal elements in Bandura's theory of self-efficacy and an anxiety-based model of avoidance behavior. J Pers Soc Psychol 1982; 42: 764-81.

[17] Feltz DL. Self-confidence and sports performance. In: Pandolf KB, Ed. Exercise and sport sciences reviews. New York: MacMillan 1988; pp. 423-457. 